involves the operation of folding and is important in the theory of almost periodic functions. The main theorem which is finally proved at the end of the lectures is that the class of almost periodic functions is identical with the class $H\{s(x)\}$ of functions which can be uniformly approximated by finite $\operatorname{sums} s(x)$ composed of terms of type $a_{n} \exp \left(i \lambda_{n} x\right)$, where the coefficients $a_{n}$ are complex quantities and the exponents $\lambda_{n}$ real quantities all of which can be chosen freely. The analysis deals largely with mean value theorems, the multiplication theorem, the uniqueness theorem and its equivalence to the Parseval relation, limiting forms of integrals, and a convergence theorem for infinite series of type $s(x)$ when the Fourier exponents are linearly independent. The report closes with an account of some generalizations of the idea of an almost periodic function. The work is all of a high standard and will stand the scrutiny of mathematicians who insist on a statement of all the saving restrictions.

H. BATEMAN

\title{
KAMKE ON PROBABILITY
}

Einführung in die Wahrscheinlichkeitstheorie. By Erich Kamke. Leipzig, S. Hirzel, 1932. vii +182 pp.

This excellent Theory of Probabiliiy is an elaboration of the author's paper Über neuere Begründungen der Wahrscheinlichkeitsrechnung.* In this paper Kamke sets forth a new foundation for probability and gives a critique of some closely related theories, those of $\mathrm{R}$. von Mises, Dörge, and Tornier. Von Mises-whose work marks the beginning of a new era in probability theorydiscards the conception of "equal likely events" as a proper basis for probability, in favor of a treatment based upon infinite sequences. If a die is thrown, and the results recorded,-for example, $2,5,3,3,6,1,4$,- - a sequence is obtained which may be thought of as continuing indefinitely. Now let $m$ be the number of times that the five-spot appears in the first $n$ throws. Some authors naively write: $\lim m / n=1 / 6$. But, in general, no such limit exists. We know nothing about the future behavior of the die. A rigorous treatment of probability must divorce itself from all physical considerations. This the newer theories do. We may write: $a_{1}, a_{2}, \cdots, a_{n}, \cdots$, as symbols for a set of numbers, restricted to the values $1,2,3,4,5$, and 6 . And among all possible sequences of this description we may choose to consider only those for which $\lim m / n=1 / 6$. And the properties of such sequences may be ascertained with mathematical rigor. Sometimes the sequence definition is called the "statistical" definition of probability. This is unfortunate, for the word "statistical" is likely to carry a reader's mind to the loose "limit" first mentioned. A sharp distinction between such a pseudo-limit and a real limit is fundamental for all clear thinking in probability. The theory of R. von Mises is grounded upon two axioms; that is, he considers only those sequences for which both axioms are valid. The first axiom relates to the existence of limits, such as has just been illustrated. The second axiom involves a selection-from the original sequence-of terms by some scheme which relates to their position or order-to the subscript $r$ of $a_{r}$

* Jahresbericht der Deutschen Mathematiker Vereinigung, vol. 42 (1932), pp. 14-27. 
and not to the value which $a_{r}$ takes on. The chief objective or this axiom II is that such a "position-selection" should yield a new sequence with the same limit values. A periodic sequence, except in trivial cases, would not satisfy Axiom II. Kamke observes, however, that the existence of sequences conforming to the two axioms of von Mises has not been established. Kamke's theory gravitates more in the direction of that of Copeland who established existence theorems for sequences selected from an original sequence by using arithmetic progressions of the subscripts. Indeed, Kamke attributes (pp. 134, 140) to Copeland two of the theorems appearing in his book; and he attributes (p. 102) to Tornier a theorem on "probability fields,"-such a field being a countable set of sequences satisfying certain conditions.

However, Kamke's approach to the subject is not the axiomatic approach. He considers, indeed, sequences for which certain limits exist; but he does not lay down at once as axioms a complete set of statements from which all further statements are to be obtained deductively. Rather, he introduces his hypotheses as they are needed.

Although the book deals here and there with biologic or economic problems (the Marbe material on the succession of male and female births (iteration or "runs," pp. 58-60); the Mendel law of heredity (pp. 70-73); the Lexis dispersion theory (pp. 161-176)) Kamke's primary interest lies in purely mathematical problems, such as the Tschebyscheff problem (p. 110): What is the probability that a common fraction will be in its lowest terms? On page 3, Kamke begins to make preparation for this problem by laying down the common fractions in a definite order. Indeed, until such a sequence is laid down, the problem has no solution - a fact overlooked by many writers who have treated this problem. A number of game problems, too, appear as illustrations.

The book consists of two parts of about equal length. The first part deals with probability-sequences, singly or in finite number; and the second part with probability-fields, countable sets of probability-sequences. A probabilitysequence is one in connection with which certain limits exist. Part I deals with two addition laws, a division law, and five multiplication laws. An exact mathematical definition of independence is used, following the lead of Hausdorff. A treatment is given of Newton's formula for repeated trials, mean value, variance, risk, and the case where an infinite set of distinguishing marks appears among the terms of the sequence. Part II deals with the existence of probability-fields with a prescribed matrix of distinguishing marks. For these marks, probabilities must exist as limits in the individual sequences, and one or the other of two multiplication laws must be valid. For these fields, then, certain multiplication laws are proved involving upper and lower limits. A Bernoulli probability-field is one satisfying the more restrictive multiplication law, and having a constant probability for the appearance of a specified mark in each individual sequence. The law of Bernoulli is proved, and the approximation formulas of Stirling, Laplace, and Poisson. By starting in turn with each of the first $k$ terms of a sequence, and annexing every $k$ th term thereafter, there is formed from a given sequence $k$ partial sequences. If for each positive integral $k$, the sequence thus formed is a Bernoulli field-so far as it goes-with constant probability, the original sequence is called a Bernoulli sequence. Not every probability sequence is a Bernoulli sequence, but for a 
given probability $w$, with $0<w<1$, a Bernoulli sequence can be constructed; in fact, for the same w, a set of sequences having the power of the continuum. The "law of large numbers" and the "fundamental law of probability" leading to the "normal" probability function and its integral are then established. The Lexis dispersion theory concludes the text proper. Then follow: a one-page 4-place probability integral table; answers to the 18 exercises given in the text; a short list of important books and papers; an index for authors and subjects.

Kamke's book is by no means an elementary text. Though it presupposes no knowledge of conventional probability material, the reader should be well grounded in analysis. Kamke regards probability, not as a mysterious field closely allied to philosophy, but simply as a branch of function-theory directed toward infinite sequences of designated types. The book is compact; but it is extremely well written, and it is also surprisingly free from typographical imperfections. In the opinion of the reviewer, this is one of the most distinctive and important treatises on probability that have appeared in recent years.

E. L. DoDd

\section{DUBISLAV ON FOUNDATIONS}

Die Philosophie der Mathematik in der Gegenwart. By Walter Dubislav. (Philosophische Forschungsberichte, Heft 13.) Berlin, Junker und Dünnhaupt Verlag, 1932. vi +88 pp. R.M. 3.80 .

Die Definition. By Walter Dubislav. Third, revised and augmented edition. (Beihefte der Erkenntnis, Heft 1.) Leipzig, Felix Meiner, 1931. viii+160 pp. R.M. 14.00 .

In the little book Die Philosophie der Mathematik in der Gegenwart, Dubislav gives an incisive discussion of certain problems of the foundations of mathematics. The problems, "What is mathematics?" and "What can it claim to prove?" are subjects of vigorous controversy; our author gives the word to all contenders, and then has his own wise comments on the views of each.

After a presentation of elements of symbolic logic - a tool essential to further progress - he devotes a section to the "metamathematical" group of problems. The very fact that the word is used indicates Dubislav's place in the camp of Hilbert. As Ramsey puts it, ${ }^{*}$ metamathematics consists of real, meaningful assertions about mathematics, which is itself meaningless; and, precisely, the main problems - those which this book deals with-are those of consistency, of the possibility of decision (general validity and fulfillability), and of completeness. The treatment in a work of this size must, of course, be fragmentary, and Dubislav chooses to emphasize the latest results obtained by the Hilbertians-for instance, Gödel's proof that all logico-mathematical calculi which comprise arithmetic contain indeterminable statements, one of which asserts the self-consistency of the system, $\dagger$ and Dubislav's own contribution to the problem of decision.

* The Foundations of Mathematics, p. 68.

$\dagger$ Kurt Gödel, Über formal unentscheidbare Sätze der Principia Mathematica und verwandter Systeme, Monatshefte für Mathematik und Physik, vol. 38 (1931), p. 173. 